\title{
DYNAMIC CHARACTERISTICS OF THE
}

\section{MOTOR COORDINATION SYSTEM IN MAN}

\author{
LAWRENCE STARK, MITSUO IIDA, and PAUL A. WILlis \\ From the Neurology Section, Yale University School of Medicine, New Haven, and \\ the Neurology Section, Massachusetts Institute of Technology, Cambridge. Dr. Iida's \\ present address is Nagoya University School of Medicine, Nagoya, Japan
}

\begin{abstract}
The dynamic characteristics of the human motor coordination system differ depending upon the physiological state. By using complex inputs the predictive apparatus is eliminated and steady state experiments can be used to study the neurological response. A series of linearity and other control experiments elucidate the limitations and adequacies of the method. Some of the features shown are a resonant peak at 3 c.P.s. and both minimum and non-minimum phase elements.
\end{abstract}

\section{INTRODUCTION}

One of our aims is to describe the human motor coordination system in quantitative terms. In addition to an adequate transfer function, we hope to derive a system model which not only fits these quantitative data but even more importantly includes anatomical, physiological, and neurological facts in order to better define the topology of the model $(4 a, b, 5,14,15)$.

The present paper is limited to a consideration of the dynamic characteristics of human motor coordination obtained with experiments carried out under restricted but well defined ranges of performance. Previous work similar to this has been done in a series of human engineering experiments recently reviewed by McRuer and Krendel (3, 6-9).

However, we are not concerned with defining human operator performance in the human engineering sense, but rather with clarifying aspects of the physiological multiple-loop control system which is in action during the human movement under investigation. For example, data obtained at higher frequencies often give most information about the equivalent networks being studied, even though these frequencies may not be very useful in terms of practical human machine-control operations 


\section{EXPERIMENTAL APPROACH}

The movement we selected to study was rotation of the wrist of an awake, cooperative human subject. The hand grasped a handle (see Fig. 1) which could only rotate, constraining the movement to pronation and supination, and which was restricted further by fixing the elbow. By arranging the axis of the forearm to be concentric with the shaft, the rotational inertia of the forearm was reduced. Wrist ro-

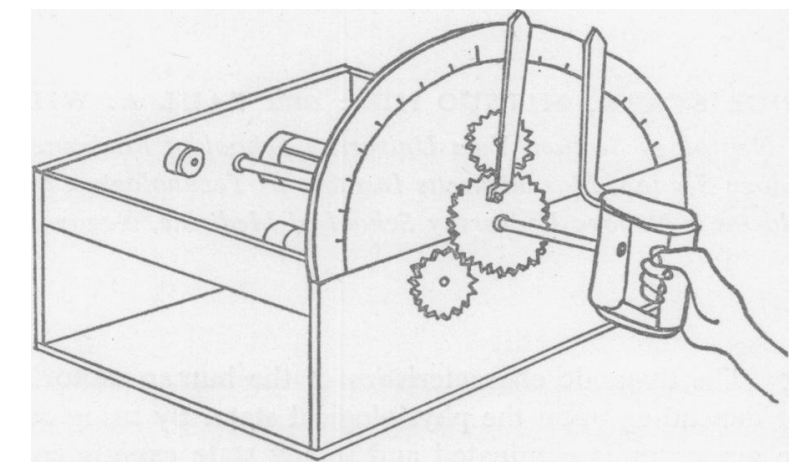

FIGURB 1 Sketch showing mechanical portion of apparatus.

tation was restricted to $\pm 30^{\circ}$ from the vertical to utilize similar muscles over the entire range of movement and to stay within comfortable limits. That these restrictions were not unrealistic is suggested by the fact that when the subject was instructed to rotate the handle back and forth as rapidly as possible, a mode of experiment called "freewheeling," the amplitude was generally confined to this range.

The input signal presented to the subject is the rotation of the pointer. The subject is instructed to keep the indicator attached to his handle in coincidence with the movement of the pointer. The instantaneous positions of both the pointer and the handle are obtained from the feedback potentiometers geared to the output shafts of their respective servomechanisms. These two signals are recorded.

In Fig. 2 is an example of a simple experiment, the predictable input experiment. Here, the input pointer swings to and fro in a sinusoidal fashion. The dynamic characteristic of the hand during a steady state predictable experiment is obtained by measuring gain and phase lag from the recordings.

Dynamic characteristics of a linear system can be described at each frequency of input signal by only two parameters, gain and phase lag. ${ }^{1}$ Gain and phase lag are defined in the usual manner; if a subject has a gain of two, while the input signal

${ }_{1}^{1}$ Schultheiss, P., and Bower, J., 1958, Introduction to the Design of Servomechanisms, New York, John Wiley and Sons, Inc.; Newton, G. C., Gould, L. A., and Kaiser, J., 1958, The Analytic Design of Linear Feedback Controls, New York, John Wiley and Sons, Inc.; Smith, O. J., 1958, Feedback Control Systems, New York, McGraw-Hill Book Co., Inc.; Blackman, R. B., and Tukey, J. W., 1958, The Measurement of Power Spectra, New York, Dover Publications, Inc. 
pointer was swinging $\pm 10^{\circ}$, then the subject's motion was $\pm 20^{\circ}$. The phase difference between two sinusoids is an angle measured in degrees; if the output follows the input by one-quarter of a cycle, then the result would be stated as a $90^{\circ}$ phase lag. Because of the variability inherent in biological systems, and particularly in man tracking a pointer, our analyses of gain and phase lag were based on the average response; that is, a number of consecutive performances were required to be essentially identical in gain and phase. The spread of phase lag did not become large except at frequencies which were on the limit of the ability of the human motor coordination system.

The importance of a small-signal approach in linearizing the responses of biological systems has been discussed in some detail previously $(10,13)$. In order to test its applicability in this instance, we have studied the effects of changing the amplitude of the input signal. (See Figs. 8 to 10.)

Complex Input. The block diagram of Fig. 3 indicates a further development of our experimental method. The input shown in Fig. 2 is completely predictable, and there is little useful information about the basic neurological control system to be obtained from it. It is therefore important to use an unpredictable input. Several methods of generating unpredictable signals are possible. One is the

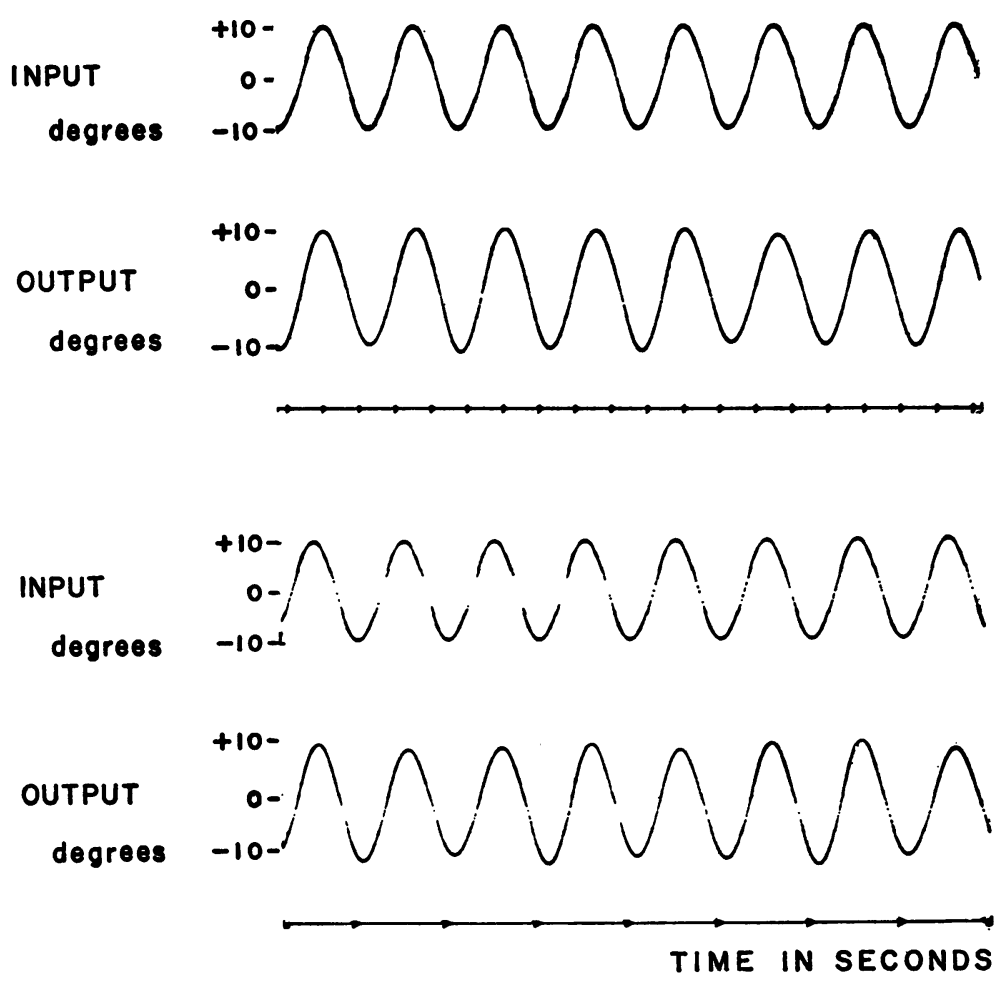

FIGURE 2 Predictable input experiment. 
use of truly stochastic noise, band-limited in a number of ways if desired, as the input signal. This, however, requires complicated computational machinery for understanding the relationship between input and output, and more sophisticated analysis instrumentation than we had available at the time. A simpler method is to sum a number of non-low-harmonically related sinusoids to form a fairly complex input signal. Of course, it is mathematically true that this signal is an analytic function and, therefore, completely predictable from its behavior in only a small neighborhood. However, this signal is not an analytic function to the prediction apparatus of the human subject operating instantaneously on the pointer movement. It only takes the sum of a small number of these sinusoids, three, if they are relatively prime to each other, in order to make the prediction apparatus of the subject inadequate. This was demonstrated in early experiments which showed no reduction in phase lag or amplitude error with considerable practice, and indeed knowledge of the structure of the input signal by the subject. Verification for this interesting physiological result is contained in the analysis section under the discussion of Fig. 13.

The principal instrumentation advantage of using a mixed sinusoidal input is that by using a narrow bandpass filter, the output can be dissected into various

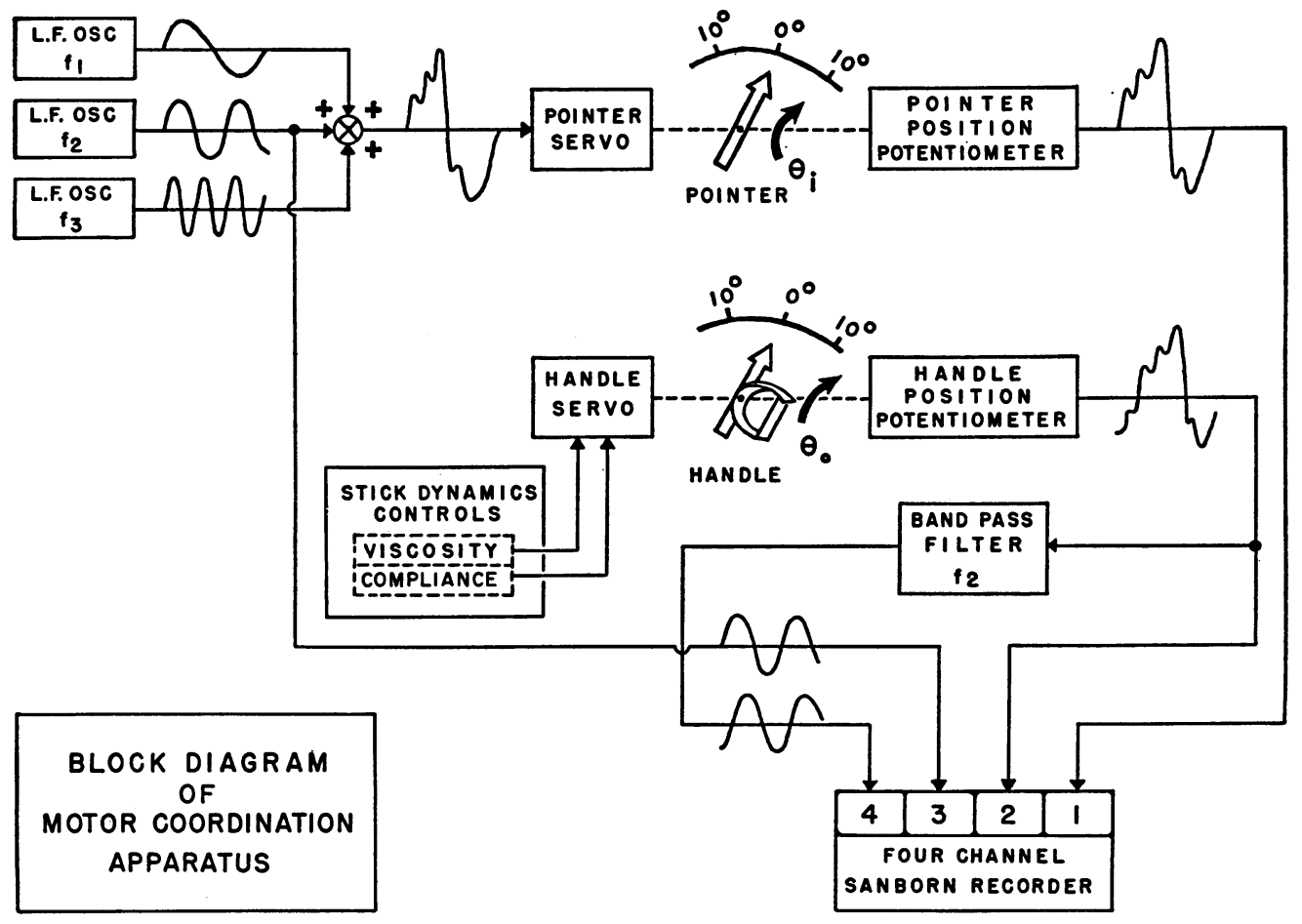

FIGURE 3 Block diagram of motor coordination apparatus. 
frequency components related to the frequency components of the input. Both the single input frequency of interest, and its dissected output counterpart were recorded, as shown in Fig. 3. The phase lag between channels three and four has two components: one due to the subject, another to the pointer servo. We determined the latter by recording the oscillator sinusoid directly and the pointer potentiometer signal through the bandpass filter. Then the handle potentiometer signal was substituted into the undisturbed filter, as shown in Fig. 3. This allowed us to subtract out the phase lag due to the pointer servo, and eliminated any possible phase lag errors due to the filter. The Krohnhite bandpass filter model 440-A was tuned to select the single frequency component of the output to be the central frequency of the input, with minus four asymptotic gain slopes occurring on either side of this frequency. These arrangements for the construction of the complex input and the dissection of the complex output enable a simple gain and phase lag analysis to be performed just as in the predictable input experiment.

Experimental Apparatus. The block diagram in Fig. 3 also indicates the operational structure of the two servo systems controlling the input pointer and the output handle. Physically, the apparatus consists of two similar 400 c.P.s. carrier servomeachanisms with concentric controlled shafts (1). Each servomechanism has a summing network, an electronic amplifier, a servomotor, and a controlled rotational element attached to the survomotor shaft. This rotational element is a pointer on one servo, and a handle on the other. Each servo has position and velocity feedback, and these two parameters as well as the gain are individually adjustable.

This ability to select control parameters of the two servomechanisms was a great aid to us. The viscosity and stiffness of the pointer servo are permanently set to achieve a slightly less than critical damping of the pointer servomechanism. In the handle servo the control of "stick dynamics" proved to be a useful part of our experimental system when, in preliminary experiments, we noted that visual tracking of the input pointer was a possible limiting factor in performance. Since this occurred at frequencies in the range 2.5 cycles per second and above and it was not our aim to test the subject's visual system, we adjusted the viscosity and stiffness of the output handle (stick dynamics) so that the response of the hand would decrease sufficiently to insure that the subject's motor system and not his vision was limiting his performance. Calibration of this equipment is performed as follows: The instantaneous positions of both pointer and handle are recorded from their respective feedback position potentiometers on the recorder. The gain of the recorder is set such that $10^{\circ}$ of pointer or handle movement is equivalent to $6 \mathrm{~mm}$. on the recording paper. This relationship is found to be linear over the range used. The dynamics of the input signal pointer servo are set to achieve an equivalent second-order system damping constant of approximately 0.6. The dynamics of the handle servomechanism were adjusted so that the subject rotated the handle against a spring force of 90 inch-ounces per radian and a viscous friction force of 1 inch-ounce-second per radian (16). The stiffness was an important factor which acted to limit the subjects response. It, however, was not frequency-dependent and therefore did not change the form of the Bode plot. The viscous friction was a much smaller factor, even though frequencydependent, and it did not become of equal importance to the stiffness until the frequency reached 15 C.P.s. (and then dominates above this frequency.) Notice that this is a much higher frequency than that of our range of interest (see Fig. 12). It thus did not con- 


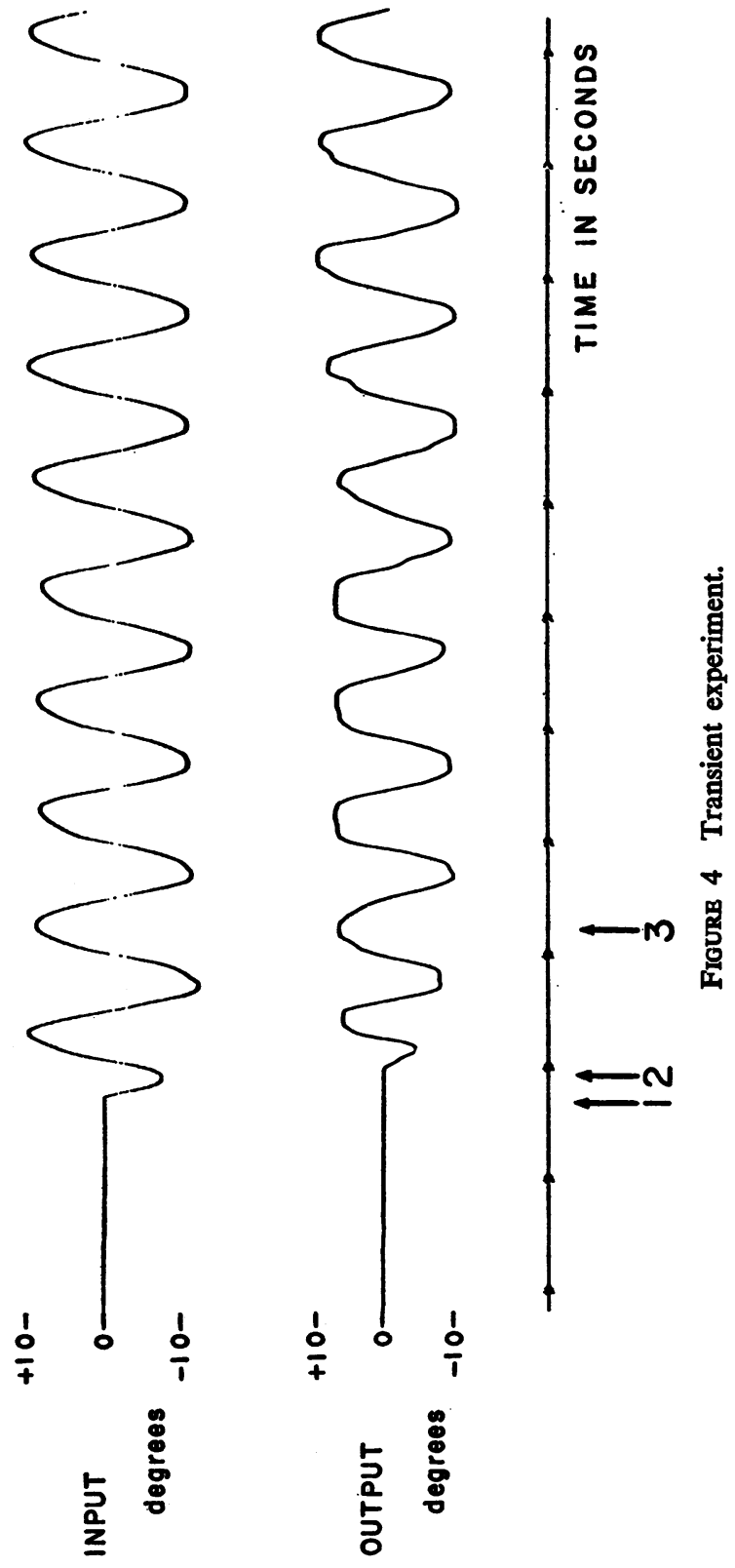




\section{INITIAL EXPERIMENTS}

tribute significant distortion to the Bode plot. Its principal use was to stabilize the handle servomechanism. The frequency responses of both servos were obtained in the usual manner, and neither limited the experiment or experimental accuracy. For the purpose of these experiments we rendered the pointer servomechanism frequency response flat out beyond the frequency range of the experiment by prewhitening the input signals.

Predictable Input and Transient Experiment. The response of a subject to a suddenly applied sinusoidal input signal from the pointer follows three successive phases of operation. In Fig. 4 such a result is shown. In the upper recording, the movement of the pointer is displayed, and on the lower recording, the movement by the subject. The first of the three phases is a reaction time delay, and is the period between numbers 1 and 2 along the time axis in Fig. 4. During this time the subject makes no movement. When the subject finally does make a movement and enters into the second, or "neurological response" phase, shown between numbers 2 and 3 along the time axis in Fig. 4, he generally starts in the proper direction to catch up with the pointer. Sometimes the initial movement of the subject is in the opposite direction, but the subject soon corrects himself and attempts to follow the pointer movement. For a variable period of time lasting approximately 0.5 to 1.0 second during this neurological response phase, there is a phase lag in the subject's following movement as well as an amplitude difference, generally resulting in a fairly sizeable error. The subject, however, soon predicts the future course of a simple sinusoidal motion and is able to phase-advance and correct his

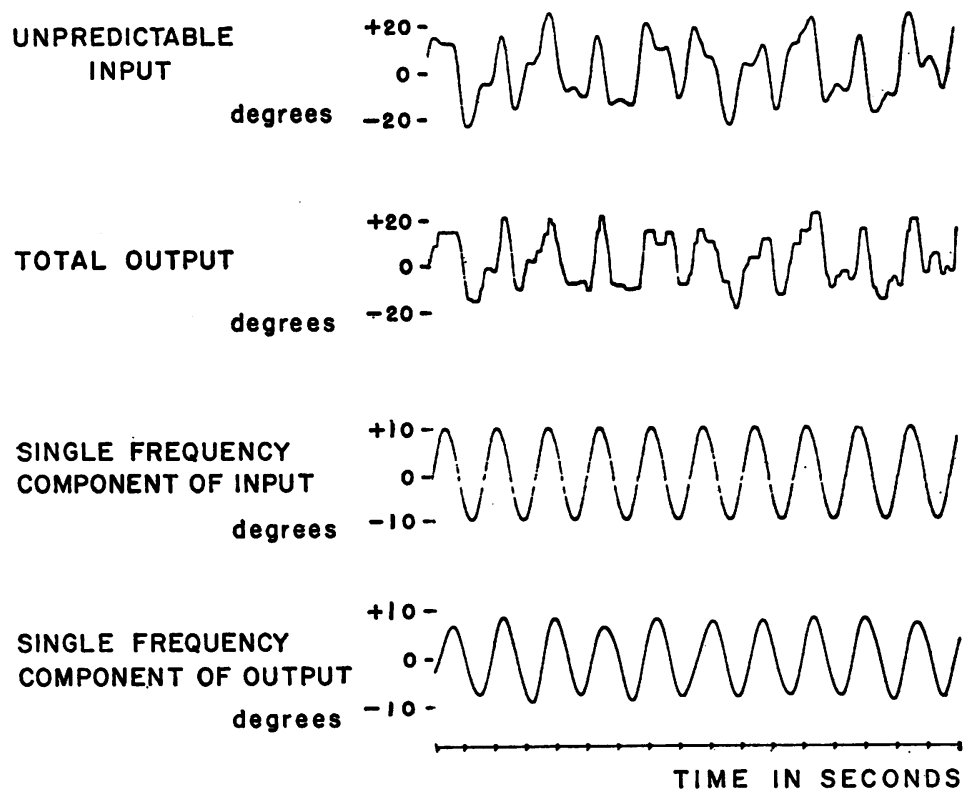

FIGURB 5 Unpredictable input experiment. 
following movement so that he eventually synchronizes his response with the input signal, as shown to the right of number 3 along the time axis in Fig. 4. It is this third or "predicted response" phase of operation which is the steady state response of a subject to a simple predictable input. In addition to the time functions shown in the third phase of Fig. 4, there are several samples of this steady state predictable following shown in Fig. 2. Rarely does it take a subject longer than a second or two to get into phase three and remain there.

Unpredictable Experiment (Fig. 5). This predicted response behavior introduces a serious and central problem in our study. In order to handle this situation, we have been forced to utilize non-predictable inputs in order that the second phase of response, the "neurological phase," is definitely prolonged. In Fig. 5, a sample experiment of the unpredictable signal input type is shown. The top recording shows the sum voltage of the three input sinusoids, as they appeared at the position feedback potentiometer of the signal input pointer. The wave form as seen by the human eye across the stretch of graph paper comprising 20 seconds of time shows certain regularities. However, to the subject who is concentrating on the motion of the pointer, and who is attempting to remain in coincidence by moving the handle-loaded with moderate viscosity and stiffness-and having only instantaneous knowledge of pointer position, the signal in the top recording appears quite unpredictable. Evidence for this physiological result is found in the analysis of the experiments (see Fig. 13).

The second recording of Fig. 5 shows the total output of the subject in following this complex input signal and it is seen to resemble the form of the input with some degree of precision.

The parts of the input and output signals which were analyzed are shown in the remainder of this figure. The third recording represents one frequency component of input obtained by recording directly the output of one of the input sinusoidal generators. The bottom recording is obtained from the total output by passing it through a narrow bandpass filter set to the single frequency of the input signal that we wish to study. Using the data obtained from the simplified input and output, we were able to determine gain and phase lag parameters between input and output exactly as in the steady state response to a predictable input. We thus have a way of preventing the prediction apparatus of the human subject from dominating the physiology of the neurological response.

Control Experiments (Figs. 6 to 10). Certain of our control experiments are necessary to demonstrate the adequacy of these methods and the difficulties involved. In Fig. 6, an experiment is shown demonstrating bandpass filter transients. In the second recording the handle movement is seen to stop suddenly when the subject is instructed to cease movement. This cessation of movement lasts for approximately 10 seconds, at which time the subject is instructed to again follow the input pointer. The complex and simple inputs continue as can be noted in the top recording and the third recording. 


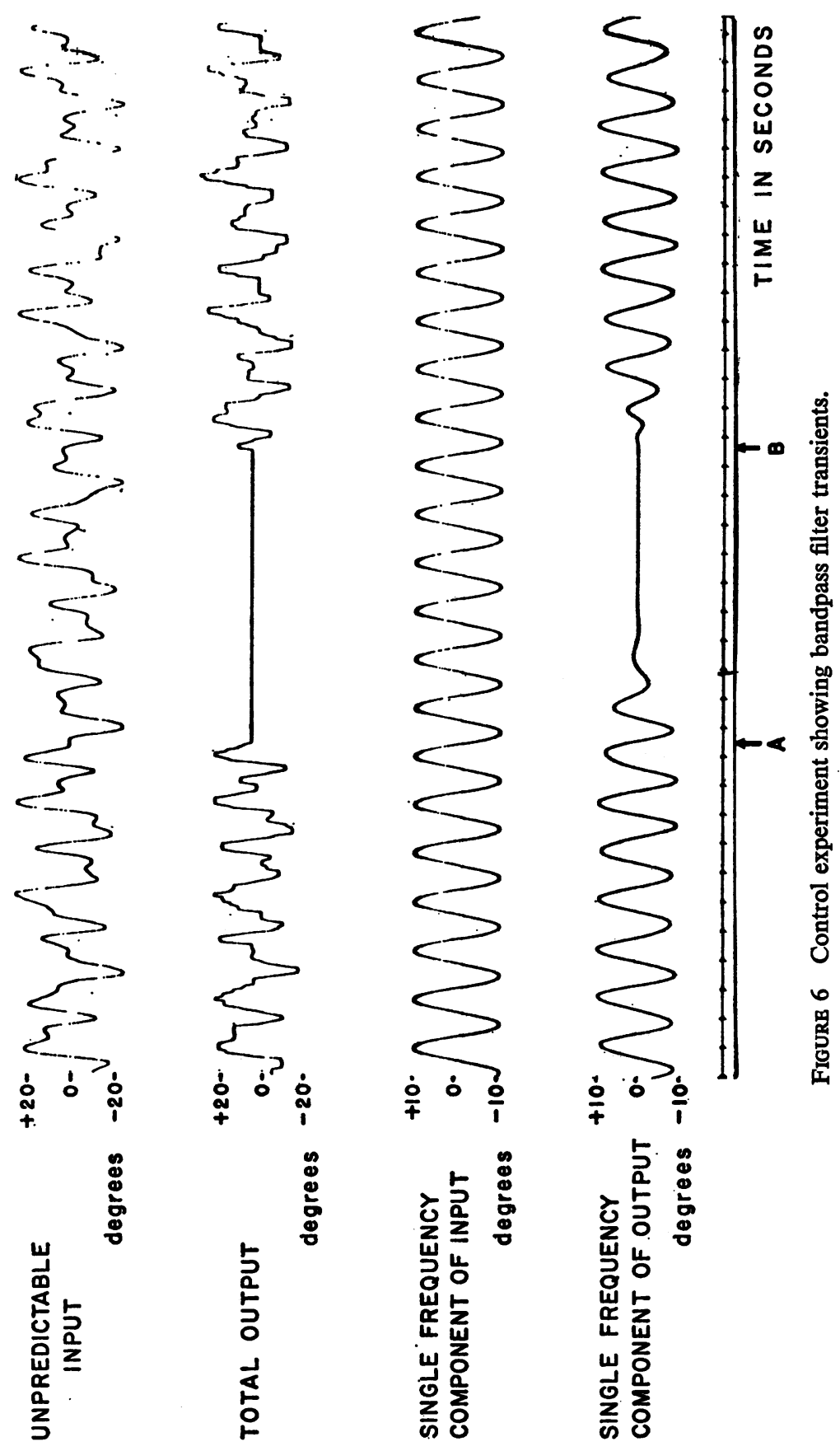


The bottom recording shows the effect of the narrow bandpass filter which we used to simplify the complex wave form. First, the filter continues to put out a diminishing wave form after the subject has ceased to move the handle. Why is this so? There are certain mathematical relationships between the time and frequency representation of a function; the narrower the bandwidth of the filter, the less quickly it can respond to a transient change of conditions. In an effort to remove contributions from other frequencies from the wave form in the lower channel, we made the bandwidth of the filter quite narrow. Therefore, it takes an appreciable amount of time (approximately 1 to 4 seconds at the frequencies we used) for the filter to reduce its amplitude as did the actual function in the total output channel. Similarly, when the subject began again to track, the filter output lagged behind the real output by several seconds. This filter action indicates the necessity for a fairly steady state response function in order to permit frequency analysis. We restricted our analysis to such sections of the experimental record. By this, we mean that we required the phase lag and amplitude of ten successive wave forms to be fairly closely grouped about their averages.

Fig. 7, noise in signal spectrum experiment, shows the efficiency of the bandpass filter in rejecting all but its central frequency. Again, the top recording, unpredictable input, shows the complex summation of three input wave forms up to the point marked "A." At this point, the single frequency component of the input, to which the bandpass filter is tuned (shown in the third recording), was set to zero. This change is reflected in both the unpredictable input and total output channel recordings. The fourth recording, the single frequency component of output, is obtained by passing the total output shown in channel two through the bandpass filter. Notice that the ringing phenomenon of the bandpass filter is apparent from point A until a relatively small noise level is reached. This noise level continues until a bit past $B$, when the frequency to which the bandpass filter is tuned is again present in both the total input and the total output. This experiment demonstrates that the so called remnant response of the subject is small in comparison with the signal swings $(8,12)$, and probably represents the effects of transient notions of the subject on the bandpass filter output. The experiment shown is representative of results obtained over many frequencies with many subjects.

Fig. 8 shows the effect of such a transient signal injected into the experimental situation. The subject responds to the superimposed step and this response contains all frequencies as a Fourier series expansion of a step function demonstrates. Only frequencies in the bandpass of the filter are not rejected. The response thus appears as a brief ringing at the central frequency of the filter. This control experiment further indicates the necessity for requiring consistent steady state relationships between input and output before accepting the data.

Another type of control experiment, and in fact an essential portion of the main experimental design, is a study of the variation in subject performance over the range of input amplitudes. We want to utilize linear system analysis; therefore we 


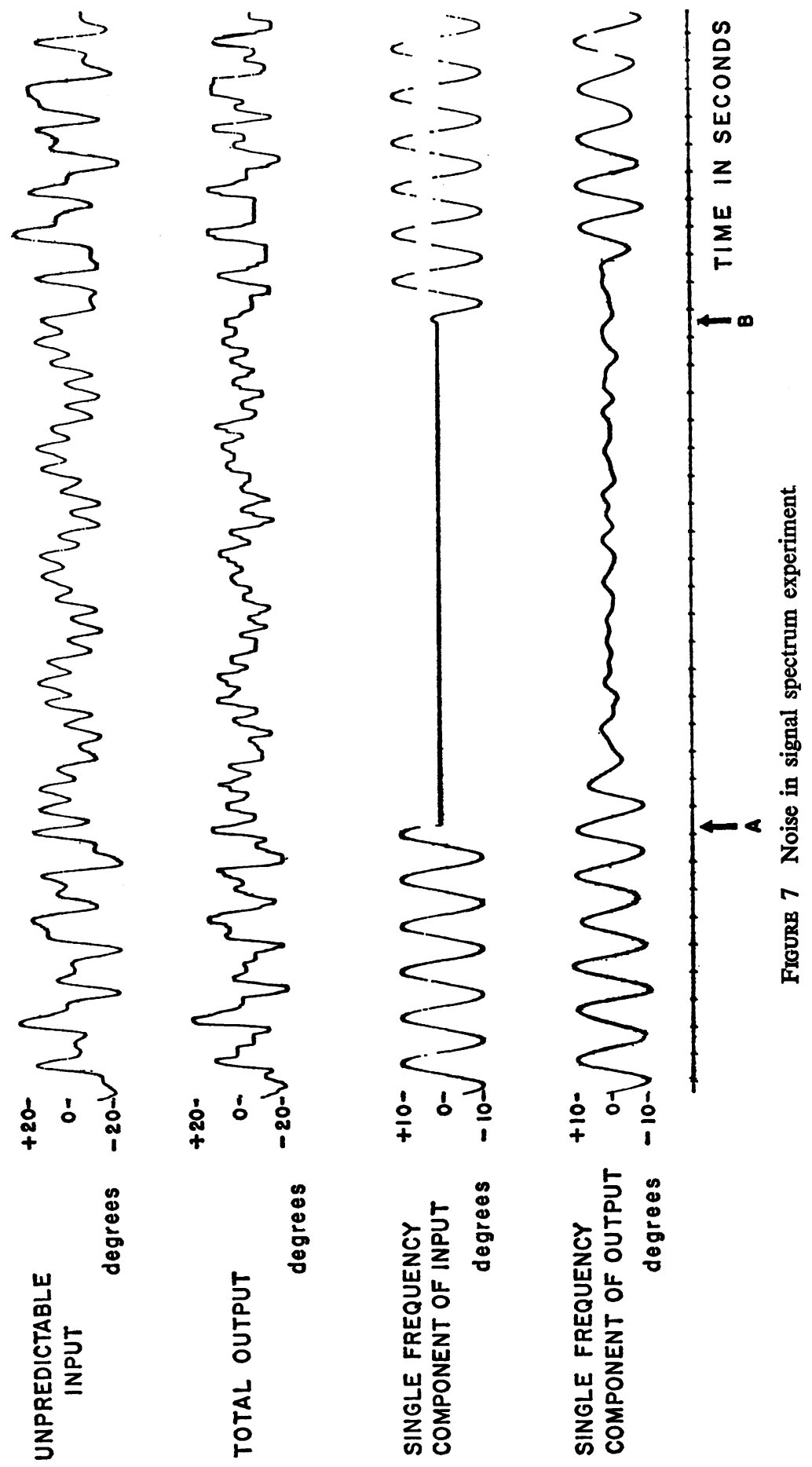




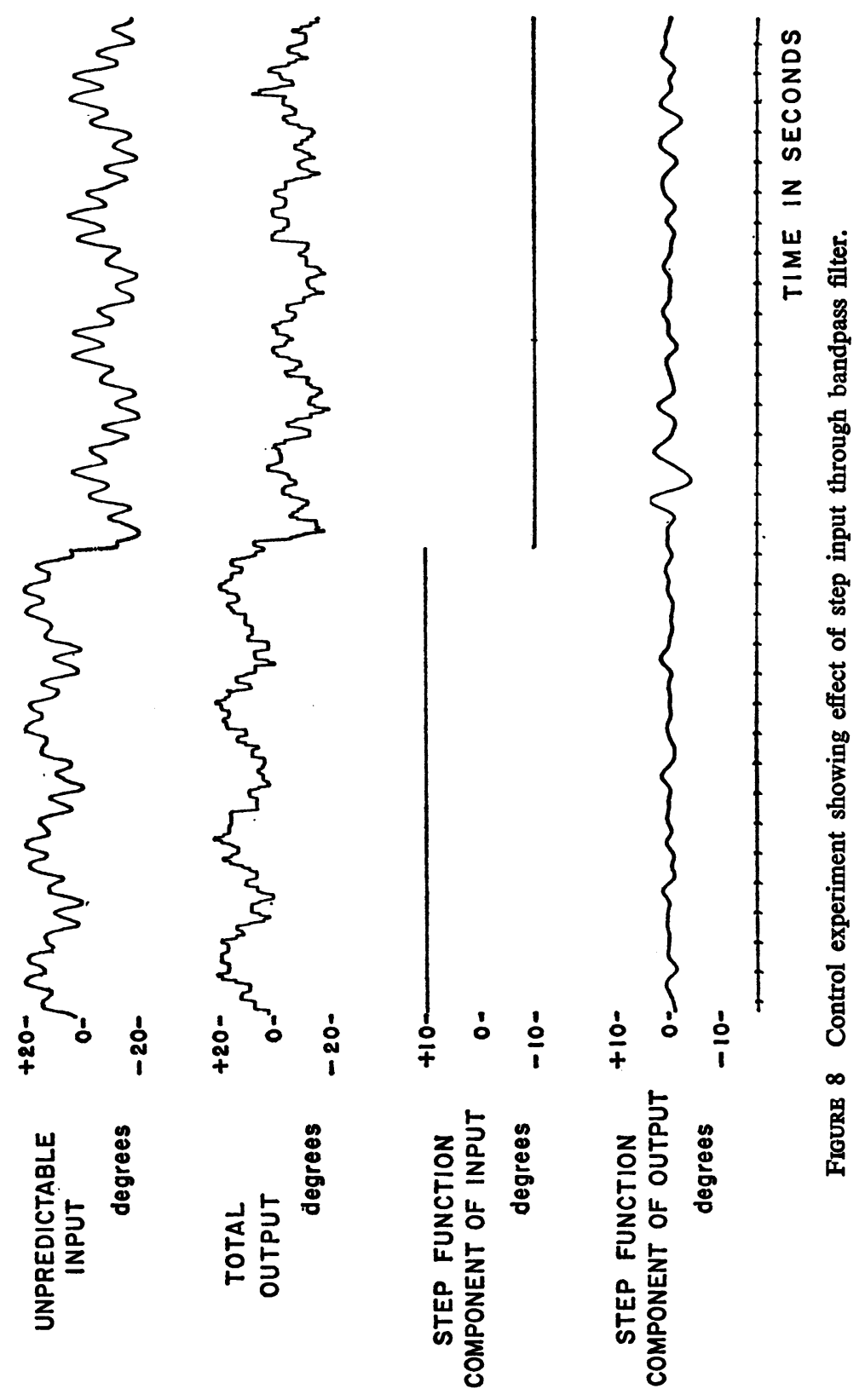




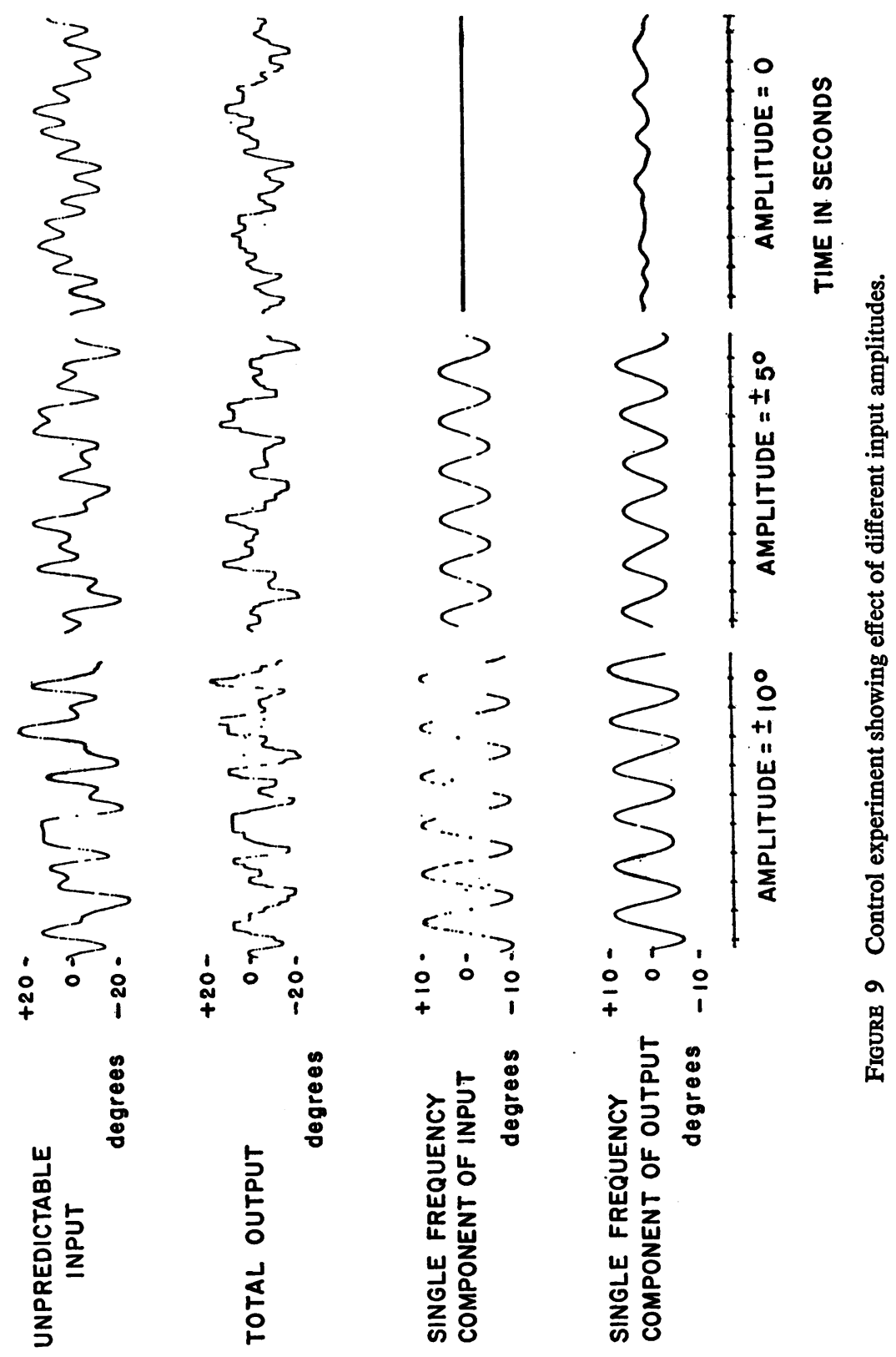


need a check on the linearity of the subject over the ranges of parameters of our experiment. As a further check on both the linearity of the experiment and the effects of the instrumentation, we performed the experiment of Fig. 9, control experiment showing effect of different input amplitudes. Again, the four recordings are labeled as they were in the previous two figures. The central frequency component of the unpredictable input was mixed at three pointer amplitude levels, $\pm 10^{\circ}, \pm 5^{\circ}$, and $0^{\circ}$; the central frequency component of the output obtained in each of these conditions is demonstrated in Fig. 9. Notice again the presence of the remnant response, due to subject motion which transiently shocks the filter. This is most apparent in the $0^{\circ}$ recording, and its amplitude is considerably smaller than the signal obtained under normal experimental conditions.

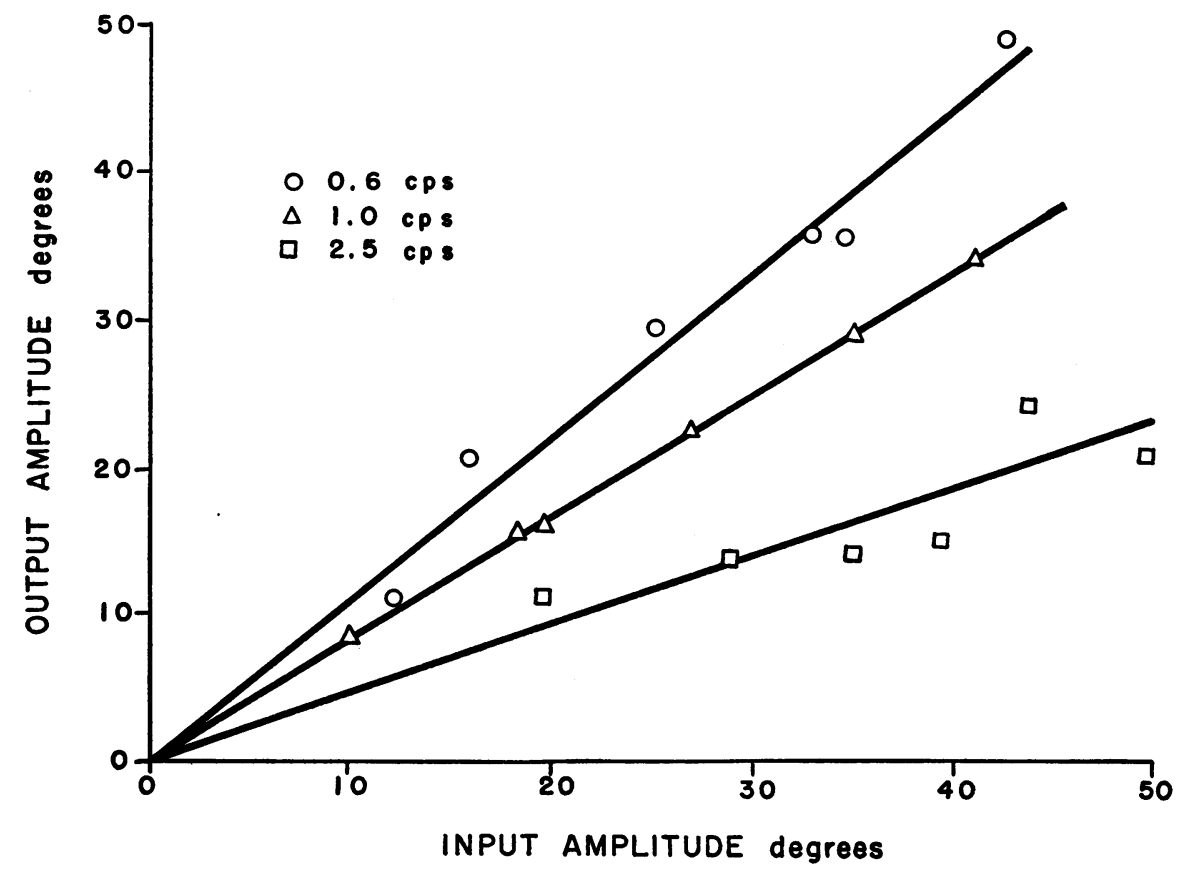

FIGURB 10 Linearity control experiment.

Experimental data to justify and substantiate that the linear approach is a feasible one are displayed in Fig. 10, linearity control experiment. This is a plot of the output amplitude in degrees versus the input amplitude in degrees, for three frequencies commonly used in our experiments. These frequencies were the central component frequencies of a complex unpredictable input for three different sets of input triads of frequencies. Notice that the subject's response at any one frequency is linear over a very wide range of input amplitude. The differences in slope values for the three frequencies are merely indicative of some of the information 


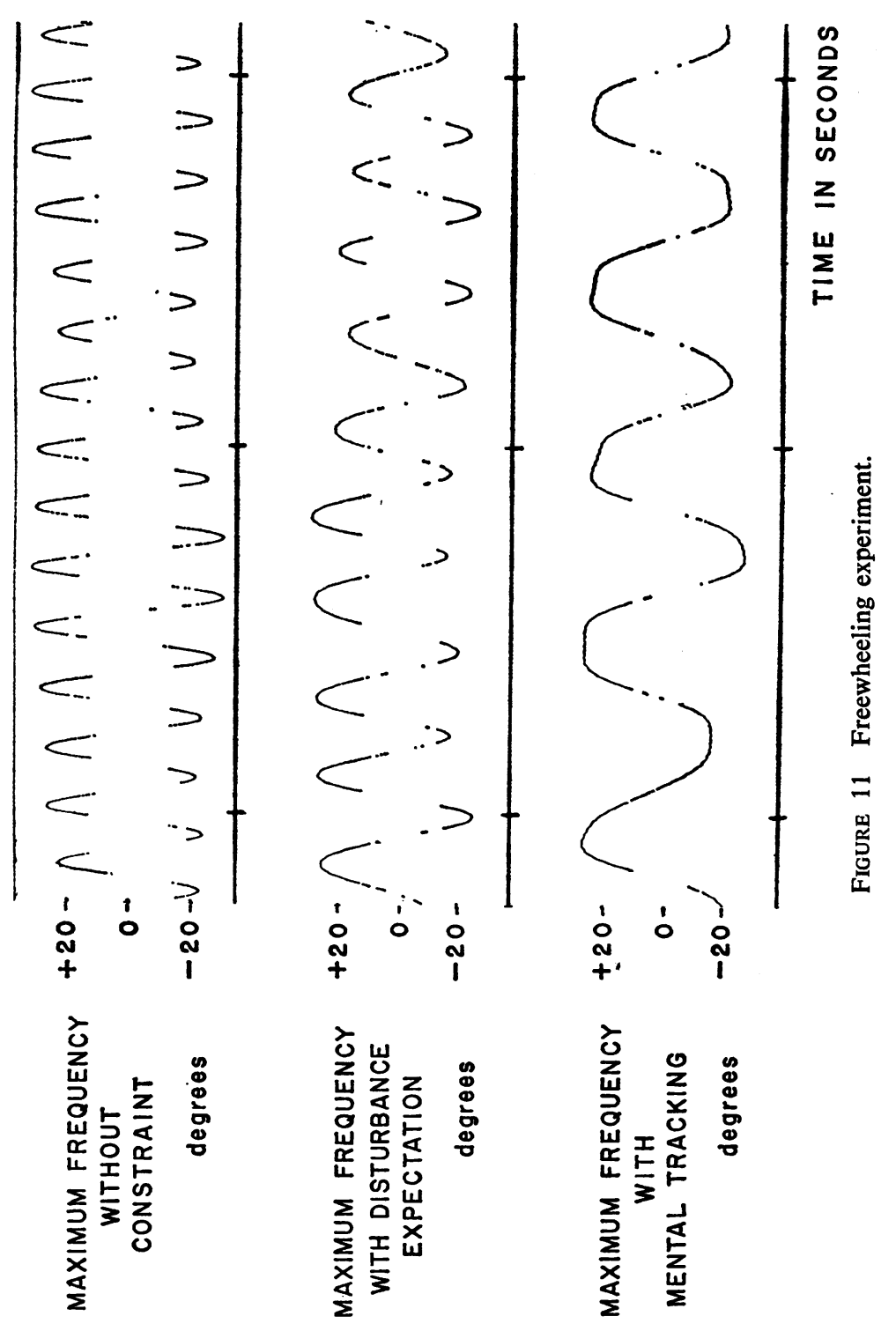


obtained in the Bode plot of the subject; it is easier for the subject to follow 0.6 C.P.S. than 1 C.P.S., and easier to follow 1 C.P.s. than 2.5 C.P.S. Hence, his gain is higher at 0.6 C.P.S. than at one of these higher frequencies. The data used in the experiment of Fig. 10 were obtained from experiments designed to eliminate trend effects. A rough estimate of the variance of this type of experiment is indicated by the scatter of the points in this figure.

Freewheeling Experiment (Fig. 11). One further type of experimental mode remains to be described. In this situation, the pointer is not used as an input signal, but the subject is asked to oscillate the handle back and forth as rapidly as possible with varying amplitudes of oscillation being requested.

Fig. 11 shows a set of freewheeling mode experiments. These illustrate an interesting fact concerning the human motor coordination system. The upper recording shows a set of oscillations with a mean frequency which is quite high. This was obtained by asking the subject to rotate the handle as fast as possible, picking a comfortable amplitude of swing. It was noted that the subject always let his muscles go quite lax when doing this experiment. This means that the system might well be operating as an open loop bang-bang control system. (2)

In the second recording, the subject was requested to oscillate as rapidly as he could, provided always that his hand was stiff enough to be able to receive a blow without being deflected very much from its course. In this condition, the subject kept his agonist and antagonist contracted against each other to a considerable degree. Naturally, this produced much slower oscillations. (This is an example of impaired performance in voluntary movements that is similar to slowness in Parkinson patients.)

The lowest recording of this figure indicates still another and very interesting operating mode. The subject is told to keep the frequency as high as possible, in the mode of the first part of the figure. However, he is instructed to imagine the pointer oscillating back and forth as fast as he can possibly track it, and is then instructed to track this imaginary pointer. Perhaps, because of the necessity to transmit and process all control signals through the imagery of the mental-tracking process, the oscillation is markedly slowed.

\section{MAIN SERIES OF EXPERIMENTS}

We have now discussed each type of experiment either as an example of an experiment type to be utilized in the main series below, or as a control experiment.

We arranged in random order a large number of frequencies and proceeded to study the responses of some fairly well trained subjects over a period of weeks. At each frequency the responses to both predictable and unpredictable inputs were obtained.

Each frequency triad of the unpredictable input was arranged so that the ratio of 
the amplitude of the central frequency component to the amplitudes of the outer frequency components was either $1.0,0.5$, or 0.0 ; corresponding to $\pm 10^{\circ}, \pm 5^{\circ}$, and $0^{\circ}$, respectively. A gain and phase lag analysis of the experiments was done immediately following each run and strict criteria, previously discussed, were adhered to in order to obtain the reproducible results described below. A set of freewheeling experiments was performed; these represent limits of the physical capacity of the hand in any type of oscillation, whether coherent with an input signal or not. Together these make up the experiment analyzed in the next section.

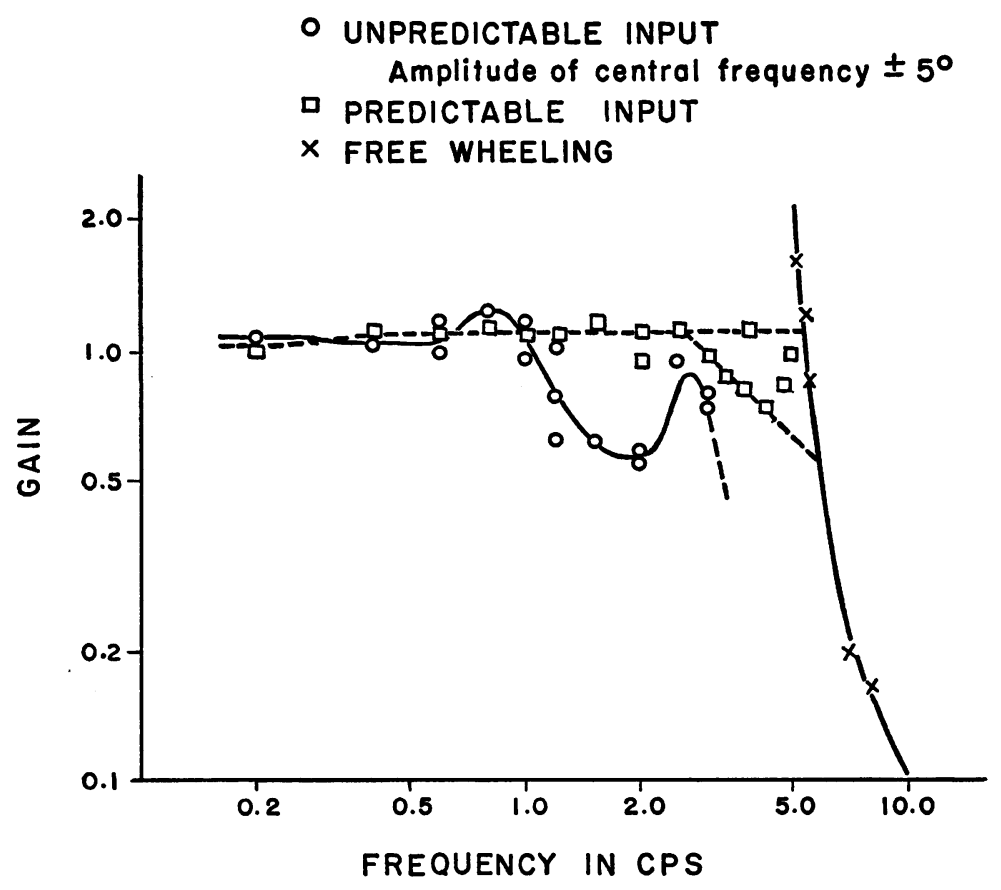

FIGURE 12 Frequency response gain data showing comparison of: (a) gain with unpredictable input (amplitude of control frequency $\left.\pm 5^{\circ}\right),(b)$ gain with predictable input, and $(c)$ relative magnitude of freewheeling.

Analysis. The basic relationships which will be displayed here are those of gain and phase lag as functions of frequency. A composite Bode plot, that is, the frequency response, of the data is shown in Fig. 12. The response to the predictable input has a gain of one for almost the entire range of frequencies studied. At approximately three cycles per second the gain decreases somewhat until the end of the range. However, the data are somewhat scattered in this high frequency end of the behavioral range of the subject.

To delineate further the actual frequency range of the mechanical behavior of the human hand in our experiment, condition one of the freewheeling experiment 
is also plotted on Fig. 12. There is, of course, no "gain" value possible for this freewheeling, because there is no physical input signal to the system; therefore, the amplitude is plotted on Fig. 12 in magnitude figures relative to the driven responses. It can be seen that an almost vertical line becomes less steep at low freewheeling oscillation amplitudes, and this line apparently represents the maximum performance of the hand in amplitude and frequeney. It was mentioned earlier that when the subject tracks a mental image of the pointer, his freewheeling frequency decreases.

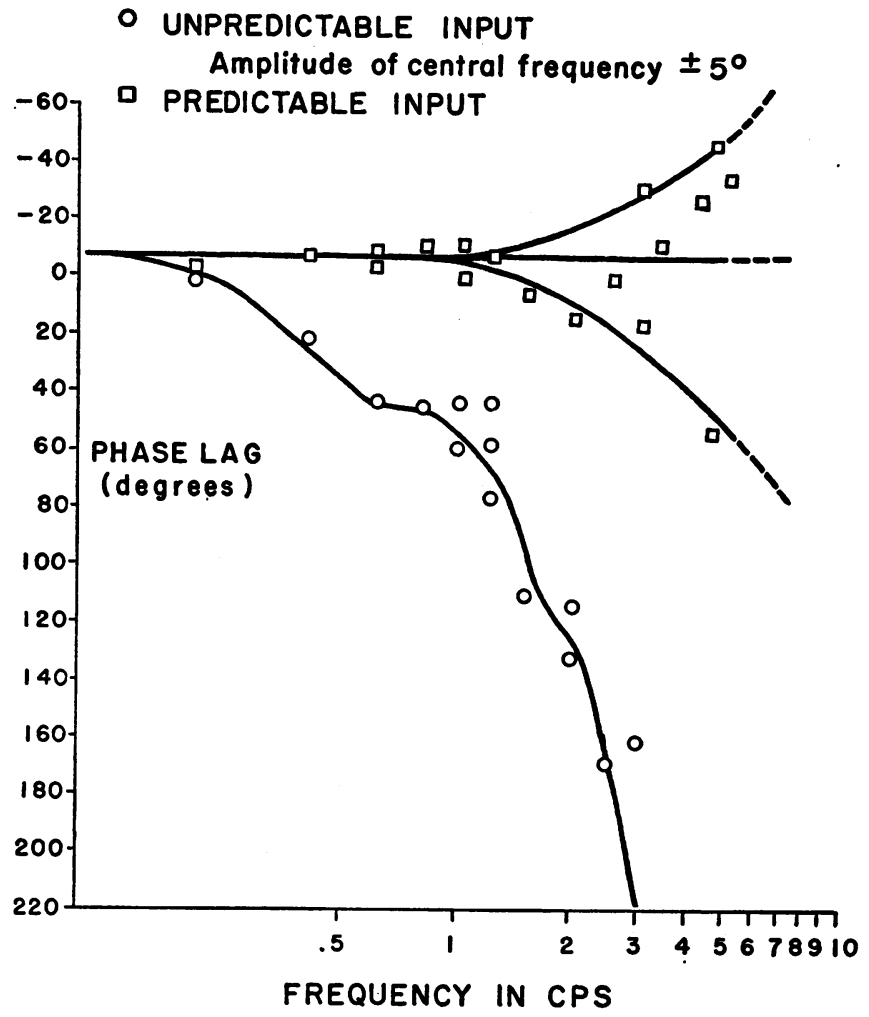

FIGURE 13 Frequency response phase data showing comparison of: (a) phase with unpredictable input and $(b)$ phase with predictable input.

It is believed that several factors may similarly reduce the response of the subject in tracking physical inputs.

The curve showing the response to an unpredicted signal over this range of frequencies is markedly different from the other two. This unpredicted or neurological response shows a gain of one up to 1 cycle per second and then a gradual reduction at a minus 1 slope with increasing frequency except for two suggestive peaks, at approximately 1 cycle per second and 3 cycles per second. There is a large area, beyond 1 C.P.S., between the gain curves of predicted and unpredicted re- 
sponses. This region represents increased performance of the system due to the prediction operation which enables the subject to correct phase and gain error in his response, and so reduce the total error.

In Fig. 13 are shown the phase data as a function of frequency corresponding to the gain data of Fig. 12. The upper curve shows the phase response to the predicted signal input. The phase changes from $0^{\circ}$ to a small amount of phase advance. Above 1 C.P.S. while the mean phase relationship continues to be one of slight phase advance, the scatter of the phase increases markedly. The cone-shaped limits at the high frequency end of the upper curve are crude variance limits of this phase behavior in the predicted experiments.

The lower curve in Fig. 13 represents the phase relationship obtained with an

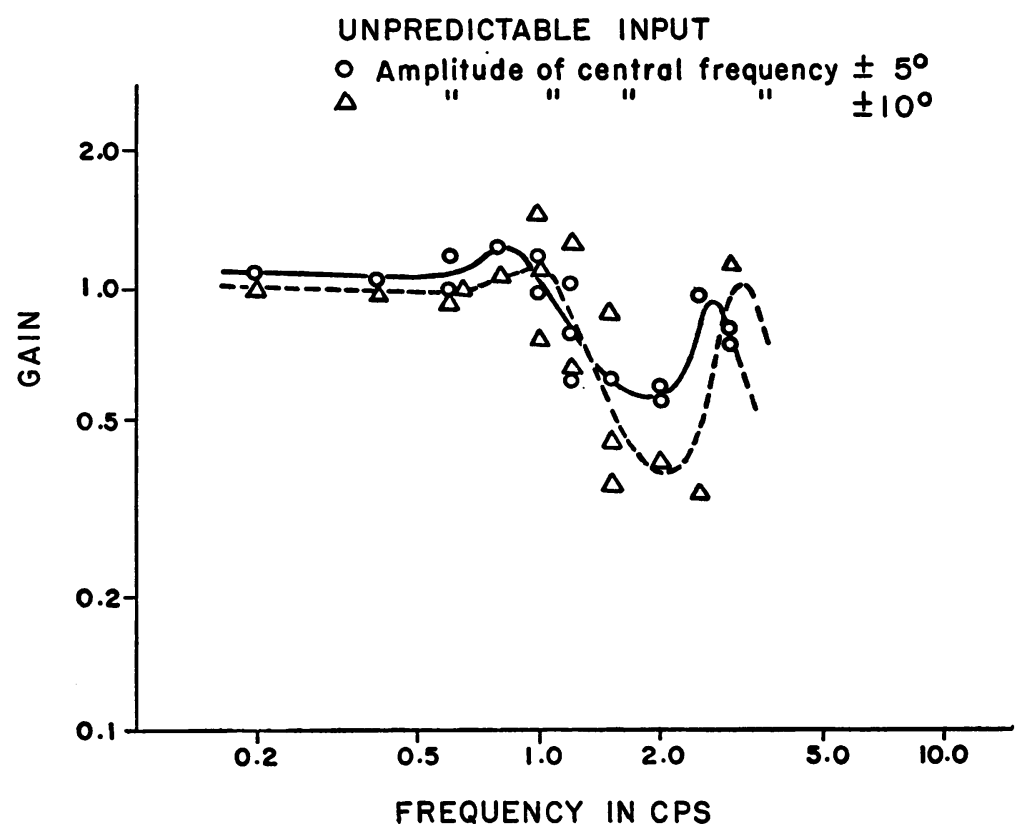

FIGURE 14 Frequency response gain data showing comparison of gain with unpredictable inputs at $\pm 5^{\circ}$ and $\pm 10^{\circ}$.

unpredictable signal input. Several interesting points are seen in this phase plot; for one, the phase lag is steadily increasing with frequency, an increase more rapid than is possible for a minimum phase lag system. This means the human servo has a non-minimum phase lag element or elements and it is possible to analyze the phase curve into non-minumum and minimum phase components. Another aspect of the phase curve is its several peaks and notches, showing irregularities corresponding to peaks of the gain curve and confirming the presence of these features in the gain curve. This is expected due to the close relationship of the gain and phase com- 
ponents of the generalized complex transfer function of a system. It is important to realize that the definite and consistent phase lags found with the "unpredictable" signal clearly show the inability of the subject to predict the complex signal.

Figs. 14 and 15 show the gain and phase plots of the unpredictable experiment for two amplitudes of the central frequency components of the input triad. The two gain and the two phase lag curves are very similar, not only in their general patterns but also in much detail. There are small but significant differences between the responses to the two amplitudes; however, these differences in the gain and in the phase curves are similar in direction. For example, the high frequency peak in the gain curve of the $\pm 5^{\circ}$ amplitude is at a lower frequency than the high frequency peak of the $\pm 10^{\circ}$ amplitude gain curve. It can be seen that the phase crossover frequency is at a lower frequency in the $\pm 5^{\circ}$ phase curve than in the $\pm 10^{\circ}$ phase curve. The above correlations show the data to be consistent within themselves and suggest that the detailed features are reliable observations. The effect of amplitude change demonstrates the presence of some non-linearities in the system. This is similar to the experiment reported by Stark and Baker wherein the transfer func-

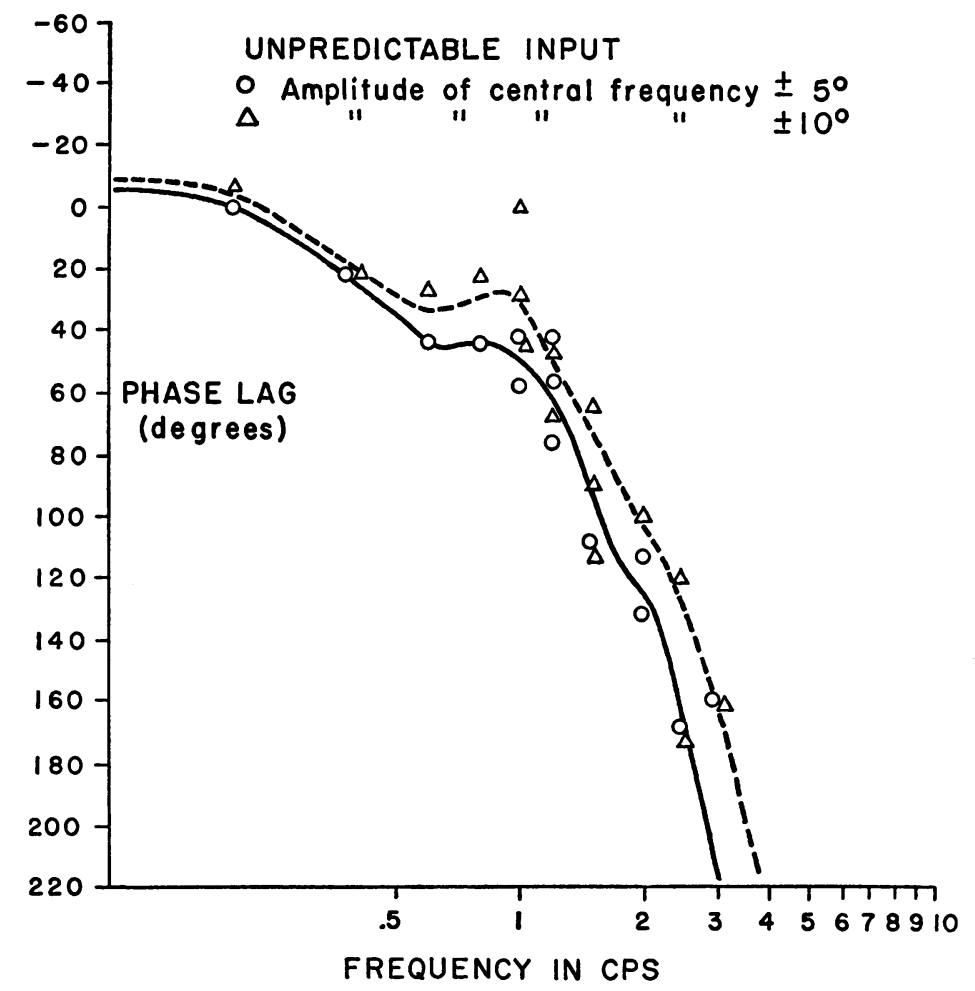

FIGURE 15 Frequency response phase data showing comparison of phase with unpredictable inputs at $\pm 5^{\circ}$ and $\pm 10^{\circ}$. 


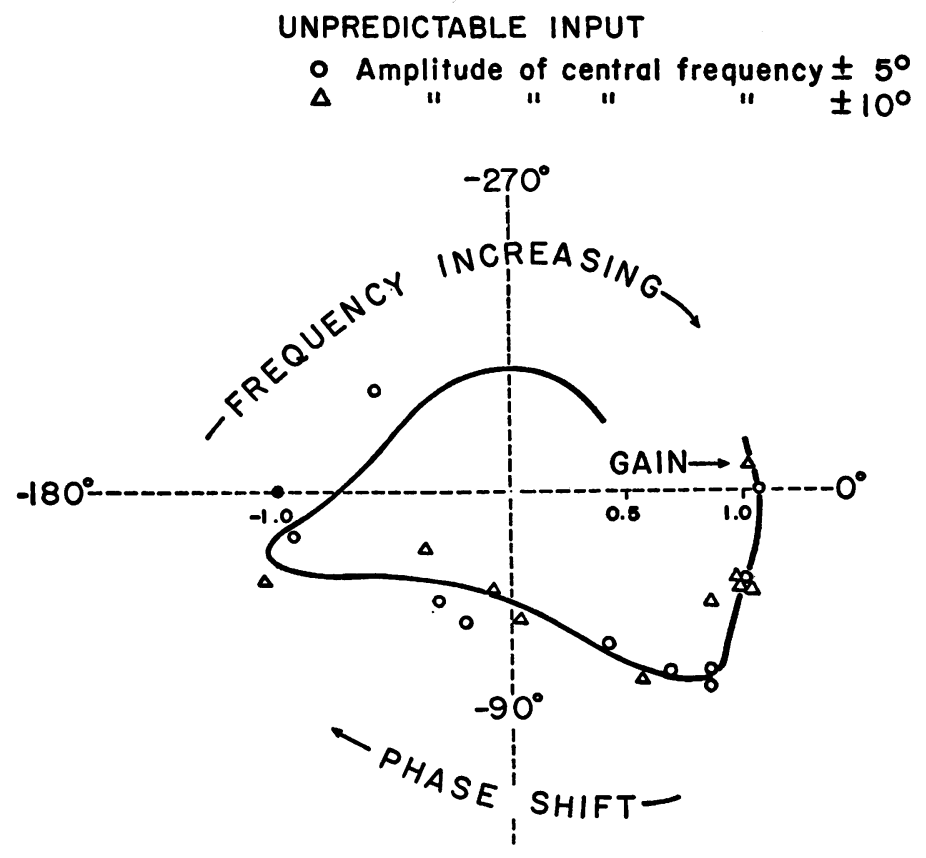

Figure 16 Nyquist diagram of closed loop unpredictable input data from Figs. 14 and 15.

tion characteristics of the pupil servo control system were changed and the frequency of the high gain oscillation which represented its resonant peak was shifted in a parallel manner (11).

In Fig. 16 is shown a polar plot of gain and phase data from the unpredictable experiment. This vector plot of gain as a function of phase lag has frequency as a monotonically increasing, but not regularly increasing function clockwise around the curve. The Nyquist curve is often used to display system behavior because of the ready availability of gain and phase stability margins of the system. This experimental curve also shows the two peaks in gain that were shown in Fig. 12.

\section{DISCUSSION}

In the Introduction we stated that this experiment was designed as a part of a complex of studies. The aim of the present paper is to describe carefully our method of obtaining quantitative information about the behavior of the control system for motor coordination and its underlying neurological servomechanisms. We have tried to give a full and careful description of the experimental methods, results, analytic methods, and faults of our present approach. We have presented evidence which shows how the prediction apparatus and the neurological response system for hu- 
man motor coordination have been separated. These aspects of the physiological system have been defined in terms of their dynamic response.

Even in looking at such crude data as we have been able to accumulate we have been impressed by the diversity of problems that are opened up by such analytic studies as described. The possibility of embedding the diverse and beautiful phenomena of human motor coordination in a sophisticated and economical mathematical representation is exciting.

This study was supported in part by United States Public Health Service Grants B-2446 and C-1196 from the Neurology and Biophysics section of the National Institutes of Health, and Office of Naval Research Contracts Nonr-609-(39), NR 144-144.

Received for publication, November 23, 1960

\section{REFERENCES}

1. Atwood, J. G., Delaney, E., and Stark, L., A transducer for testing motor coordination, unpublished data.

2. CoRTes, A., 1958, The human servo: Arm control, Master of Science thesis, University of California, Engineering library, Berkeley.

3. ElKIND, J. I., April, 1956, Characteristics of simple manual control systems, Lincoln Laboratory. Massachusetts Institute of Technology, Techn. Rep. No.111.

4a. Granit, R., and Stark, L., Unpublished experiments. 4b, GRANIT, R., 1958, Neuromuscular interaction on postural tone of cat's isometric soleus muscle, J. Physiol., 143, 387.

5. Hammond, P. H., Merton, P. A., and Sutton, G. G., 1956, Nervous gradation of muscular contraction, Brit. Med. Bul., 12, 214.

6. Johnson, A., 1959, The servoanalysis of postural reflexes, Doctoral Thesis, Massachusetts Institute of Technology.

7. KRENDEL, E. S., and MCRUER, D. T., 1960, A servomechanisms approach to skill development, J. Franklin Inst., 289, 24.

8. MCRUER, D. T., and KRENDEL, E. S., 1959, The human operator as a servosystem element, J. Franklin Inst., 267, 1.

9. Sheridan, T. B., 1960, Experimental analysis of time-variation of the human operator's transfer function, Internat. Fed. Automatic Control Congr., 1881.

10. Stark, L., 1959, Stability, oscillations, and noise in the human pupil servomechanism, Proc. IRE, 47, 1925.

11. StARK, L., and BAKER, F., 1959, Stability and oscillations in a neurological servomechanism, J. Neurophysiol., 22, 156.

12. Stark, L., Campbell, P. W., and Atwood, J., 1958, Pupil unrest: an example of noise in a biological servomechanism, Nature, 182, 867.

13. Stark, L., and Sherman, P. M., 1957, A servoanalytic study of the consensual pupil reflex to light, J. Neurophysiol., $20,17$.

14. WIENER, N., 1958, Cybernetics, New York, John Wiley and Sons, Inc.

15. WIIKIE, D. R., 1950, The relation between force and velocity in human muscle, J. Physiol., 110, 249.

16. WIILIS, P. A., 1960, Mechanical output impedance of motor coordination transducer, unpublished data. 